\title{
Similar-orientation McCollough effect and the classical negative afterimage in a common adaptation process
}

\author{
S. H. MAHMUD \\ University of Düsseldorf, Düsseldorf, West Germany
}

(Charles W. Eriksen, Sponsor)

\begin{abstract}
The McCollough effect was obtained using red and green gratings of similar orientation and spatial frequency with the subjects fixating during adaptation and test. The McCollough effect was again produced, along with the classical negative afterimage, in a single-adaptation sequence. The results show that the two aftereffects stem from different mechanisms in the visual system and that a common retinal area can be involved in both types of effect.
\end{abstract}

McCollough (1965) demonstrated a long-lasting color aftereffect by adapting subjects to alternating vertical orange gratings and horizontal blue gratings for 2-4 $\mathrm{min}$. Following adaptation, subjects viewed an achromatic test pattern containing side-by-side horizontal and vertical gratings. Subjects reported a bluish coloration on the side of the test pattern containing vertical gratings and an orange coloration on the side containing horizontal gratings.

Although the McCollough effect was obtained by adapting subjects to two different spatial orientations (i.e., vertical and horizontal gratings), differential color aftereffects of the McCollough type also have been reported following adaptation to gratings of the same orientation but of different spatial frequencies (Breitmeyer \& Cooper, 1972; Lovegrove \& Over, 1972; Stromeyer, 1972). These results may be expected if the McCollough effect is basically dependent on the tendency during natural vision to fixate on a bright stripe, thus maintaining fixed patterns of stimulation on the retina during viewing of gratings. Superimposition of the gratings of two spatial frequencies of the same orientation lead to regular differential patterns of the stimulation on the retina, provided at least one type of grating is narrower than the other. If the gratings are of identical spatial frequencies, differential stimulation of different areas on the retina is not achieved unless the adapting gratings are interlaced on the projection screen and fixation is maintained by the subject, or, in other words, unless a phase difference of half cycle exists between the two patterns. Color aftereffect may be due to the phase shift and may depend on the position of the achromatic gratings in the test pattern relative to the fixation point on either the bright or the dark stripe.

The author is now at the Department of Psychology, University of Dhaka, Dhaka-2, Bangladesh.

\section{EXPERIMENT 1}

The aim in Experiment 1 was to produce differentially colored aftereffects using adaptation gratings of identical orientation and spatial frequencies. The subject's fixation point was adjusted so that the red and green stripes were interlaced in the retinal image.

\section{Method}

Subjects. Eight male and 7 female university students served as subjects in this experiment. All had normal color vision on the Ishihara test. None had taken part in other experiments on the McCollough effect.

Apparatus and Materials. Two identical $5 \times 5 \mathrm{~cm}$ slides, each containing 38 alternating black and white stripes (1:1 ratio), were projected from a distance of $2.4 \mathrm{~m}$, giving visual angle of $15.2^{\circ} \times 15.2^{\circ}$. The frequency of the gratings was $1.25 \mathrm{cpd}$. The projectors were initially adjusted so that the black stripes of one slide interlaced with the white stripes of the other, or, in other words, so that a phase difference of half cycle between the two patterns resulted $\left(M_{1}\right.$ and $M_{2}$ of Figure 1). One pattern was projected through a red filter (Kodak Wratten No. 25) and the other through a green filter (Kodak Wratten No. 58).

A fixation point was affixed to the center of the viewing screen so that it appeared in the middle of a green stripe for $\mathbf{8}$ subjects and in the middle of a red stripe for the remaining 7 .

Screen luminance with the infiltered gratings was adjusted to $0.8 \mathrm{~mL}$ (measured with an S.E.I. spot photometer). Luminance of the red, green, and neutral areas calculated from the filter values were $0.8,0.16$, and $0.17 \mathrm{~mL}$, respectively. Low values were employed in order to create conditions that were not favorable for evoking the negative afterimage.

Procedure. Subjects were adapted for $3 \mathrm{~min}$, during which a set of red vertical gratings was alternated every $10 \mathrm{sec}$ with a set of green vertical gratings. Subjects were provided with a fixation point on which to fixate throughout the experiment. Following adaptation, subjects viewed the achromatic vertical gratings having width identical to that of the adapting pattern in such a manner that (1) the fixation point appeared in the middle of a white stripe or a black stripe, coinciding with the location of the point in the stimulus pattern, and (2) the fixation point was then moved to a series of positions corresponding to the center of either a white or black stripe in the projected image. Subjects' reports of colors experienced were obtained. Subjects were asked to report the stripe's color perceived in terms of red, green, blue, yellow, a combination of these, or achromatic. The first test was started at the end of adaptation and was completed in under 5 min (immediate test). Sub- 
jects were tested again after an interval of $30 \mathrm{~min}$ (delayed test). Four subjects were also tested at longer intervals $(50-70 \mathrm{~h})$.

\section{Results}

When fixating on the achromatic test pattern, all subjects (except 1 subject who could not report the red effect) reported complementary color effects in each test, dependent on whether the point of fixation was on a dark or a light stripe. When the fixation point was moved, the color effect was reported to have changed in an appropriate direction. For example, when the fixation point appeared on the black stripe, subjects reported a red aftereffect, and when the point appeared on the white stripe (resulting in a change in the position of the fixation point), subjects reported a green aftereffect.

All subjects reported the color effects in both immediate and delayed test conditions. Color aftereffects were also reported by the 4 subjects who were tested after 50-70 $\mathrm{h}$. It may be argued that the color effects observed in the above study were due to the formation of a negative afterimage dependent on the adaptation in specific areas of the retina. Experiment 2 was therefore conducted to determine whether separate color effects could be produced with the classical negative afterimages following a common adaptation sequence.

\section{EXPERIMENT 2}

Experiment 2 was designed to determine whether the two types of effects-the classical negative afterimage and the McCollough effect with gratings of the same orientation-arise independently in the same eye and with stimulation of similar areas of the retina.

\section{Method \\ Subjects. Volunteer subjects were 8 males and 4 females with nor- mal color vision on the Ishihara test. \\ Apparatus and Materials. Two types of adaptation pattern were used. One type was homogeneous half squares $\left(7.6^{\circ} \times 15.2^{\circ}\right)$ that were in- tended to evoke afterimages. One homogeneous field was projected through a red filter and the other field through a green filter $\left(N_{1}\right.$ and of $\mathrm{N}_{2}$ of Figure 1). Interlacing vertical red and green stripes also were employed, as in Experiment 1, and were intended to evoke McCollough- type effects $\left(M_{1}\right.$ and $M_{2}$ of Figure 1$)$. \\ Procedure. The two sets of adaptation pattern, homogeneous and striped, were projected for $6 \mathrm{~min}$ in a regular sequence: $M_{1}, N_{1}, M_{2}$, $\mathbf{N}_{2}$. During adaptation, the subject fixated at a point appearing on a colored stripe (either red or green) in the case of gratings and at the center of the homogeneous half-square patterns (Figure 1). Following adaptation, subjects were tested at intervals of $1,10,20$, and $40 \mathrm{~min}$ on the two types of patterns (fixation point on dark or on light stripe) to evoke the afterimage and to evoke the McCollough effect (Figure 1, $\mathrm{MT}_{1}$ and $\mathrm{MT}_{2}$ ). Each of these patterns was projected for $2 \mathrm{sec}$ (to avoid readaptation), and the subjects reported the color experienced.}

\section{Results}

The two types of effects were obtained independently by all subjects, according to the test pattern viewed. In the case of the classical negative afterimage, half of the test patterns appeared uniformly pink and the other half
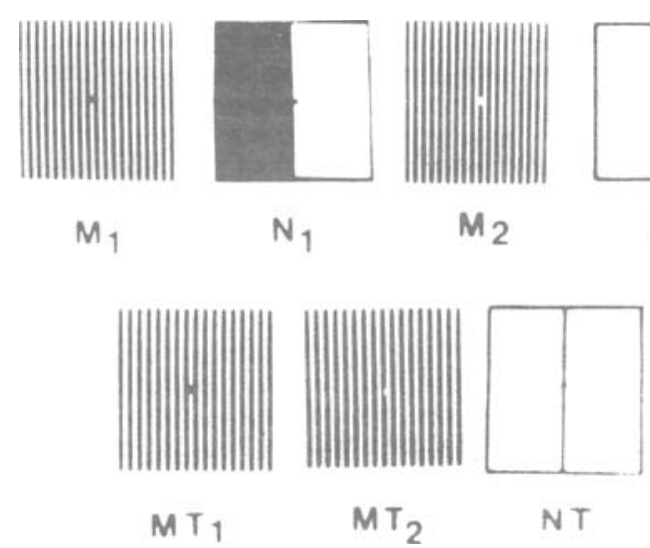

Figure 1. Patterns employed for obtaining the McCollough effect $\left(M_{1}, M_{2}, M T_{1}, M T_{2}\right)$ and the negative afterimage $\left(N_{1}, N_{2}, N T\right)$.

green when the homogeneous test pattern was employed (Figure 1, NT). Differential color aftereffects of the McCollough type were obtained when the achromatic vertical gratings were projected: the color reported was dependent on the position of the gratings in relation to the fixation point. Subjects reporting color for each test pattern are shown in Table 1.

\section{GENERAL DISCUSSION}

It appears that the McCollough effect can be obtained by employing stimulus patterns of the same spatial frequency, provided that accurate fixation can be maintained in the retinal area. The study further demonstrated that the classical negative afterimage and the McCollough effect stem from separate mechanisms in the visual system since they can be established simultaneously and evoked separately within the same retinal region. The long-term retention of the classical negative afterimage observed in these experiments is similar to that of the McCollough effect. It may be argued that the long-lasting component of the classical negative afterimage is in fact due to a McCollough effect, since the adaptation pattern consists essentially of very broad interlaced side-by-side gratings with fixation at the central dividing line. If so, based on the results of Experiment 2, it would be necessary for two separate McCollough effects, in each of which red and green stripes are seen, to be obtained independently from the same retinal area by employing stripe pairs of different spatial frequencies. An additional test has been made in which the shape of the adapting patterns has been changed. These two homogeneous half squares were replaced by two patterns of circular homogeneous colored area. These were then employed in conjunction with two vertical gratings, and similar aftereffects were obtained. These results confirm the findings of Hansel and Jones (1982) and fur-

Table 1

Number of Subjects Reporting Each Color in the McCollough Effect and the Negative Afterimage at Different Intervals

\begin{tabular}{cccccc}
\hline \multirow{2}{*}{$\begin{array}{c}\text { Retention Time } \\
\text { (Minutes) }\end{array}$} & \multicolumn{2}{c}{ McCollough Effect } & & \multicolumn{2}{c}{ Negative Afterimage } \\
\cline { 2 - 3 } \cline { 5 - 6 } & Red & Green & & Red & Green \\
\hline 1 & 12 & 12 & 12 & 10 \\
10 & 12 & 12 & 12 & 10 \\
20 & 12 & 11 & 11 & 10 \\
40 & 12 & 10 & 10 & 10 \\
\hline
\end{tabular}

Note- $N=12$. 
ther indicate that long-term aftereffects for homogeneous areas are independent of the McCollough effect or of edges or gradients in the adapting and test patterns.

\section{REFERENCES}

Breitmeyer, B. G., \& CoOPER, L. A. (1972). Frequency-specific color adaptation in the human visual system. Perception \& Psychophysics, 11, 95-96.

HANSEL, C. E. M., \& JoNES, R. A. (1982). Long-term retention of negative afterimages. IRCS Medical Science, 10, 7-8.
LOVEgrove, W. J., \& OvER, R. (1972). Color adaptation of spatial frequency detectors in the human visual system. Science, 176, 541-543. McCollough, C. (1965). Color adaptation of edge detectors in the human visual system. Science, 149, 1115-1116.

STROMEYER, C. F. (1972). Edge contingent color aftereffects: Spatial frequency specificity. Vision Research, 12, 717-732.

(Manuscript received for publication April 8, 1987.) 